\title{
IS EVERY PLAYING WITH IMAGINATION A THOUGHT EXPERIMENT?
}

\author{
Nina D. Stanković* and Vladimir M. Ristić \\ Department of Physics, Faculty of Science, University of Kragujevac, \\ 34000 Kragujevac, Republic of Serbia \\ E-mail: ninastankovic@live.com
}

(Received March 30, 2016)

\begin{abstract}
In this paper we are analyzing thought experiments that could be found in Einstein and Galileo's work, noticing that some researchers are even declaring certain presocratic thinking as thought experiments. The term was coined before Einstein, and there were others who designed experiments full of imagination. Yet thought experiments are a subset of scientific experiments and should be looked for in experimental sciences like physics, so here imaginative exercises are not accepted as thought experiments. Thus in this paper a new working definition of a thought experiment is proposed and some rather dubious examples that are now widely being proclaimed for thought experiments are properly classified. Also, we will give a general characterization of thought experiments as they appear in modern physics, to draw some conclusions about them and then analyze some Einstein's work.
\end{abstract}

\section{INTRODUCTION}

The notion of 'thought experiment' has proved to be one of the great achievements of science and education. Because it was designed in mind, it skillfully avoids the need for the elaborate laboratories and bloated financial plan of experimental physics. From thought experiments we learn about our physical world. In this paper our purpose, however, is to argue that thought experiments produce such epistemic wonders. (NORTON, 1991)

Einstein asked himself when he was young: "How the world would look like to a man if he could travel with the velocity of light?" (RESCHER, 1991). Answer can be found in his special theory of relativity. The answer is that such an accomplishment, of course, is not possible.

Yet the aforementioned question has been setting something that is not possible to achieve in natural circumstances, but would be very interesting if it could be achieved. Can we consider this a thought experiment?

Although thought experiments, at first glance, seem a mere intellectual game and as inferior compared to other methods of gaining scientific knowledge (real experiments, observation of natural processes, for instance), they still have a significant place as a research method in physics. In order to decide can this Einstein's "imaginative journey" be considered a thought experiment, we need to examine some of the more famous thought experiments: Einstein's elevator and train experiments, for instance. Theory of relativity (EINSTEIN, 1917) imposes a lot of non-standard thinking to people who study it. Teaching experience shows us 
that the most appropriate tools for accepting that unusual approach are thought experiments, most of them proposed by Einstein. Still any researcher interested in thought experiments must go far back into the past. Most of them discover thought experiments in Galileo's work (rightfully, to our opinion), but some are even proclaiming presocratic thinkers, for thought experimenters. But we, together with researchers like (SORENSEN 1992) think that thought experiments are a subset of real experiments, and they should be exclusively looked for in experimental sciences (physics, before all, because there are no thought experiments in other sciences).

But the problem lies in a lack of a useful operative definition (BABOVIĆ et al. 1983) of a thought experiment, so we are offering one in this paper. Also the precise classification (BABOVIĆ et al. 1983) of thought experiments is proposed, which suggests a thought experiment should be capable of proving the scientific statements (that is different, from claiming, like NORTON (1991) and various others do, that thought experiments are arguments). This last proposition, of course, can be questioned, so in paper (RISTIĆ RADULOVIĆ, 2001) a broader foundation for it has been given, by comparing thought experiments with computer experiments, and here a few of the conclusions are repeated, thoroughly revised.

This will lead to elimination of some widely accepted plays with imagination from the collection of thought experiments while it will also be shown that even the greatest can make mistakes and overlooks.

\section{FEW HISTORICAL REMARKS ON THOUGHT EXPERIMENT}

Hans Christian Ørsted coined the mixed term Tankeenexperiment (lit. thought experiment) circa 1812. Much later, Ernest Mach used that term exclusively to denote the imaginary conduct of a real experiment, which then would be performed by his students. Yet those, according to our classification, were not thought experiments - this kind should be called imaginary experiment (BABOVIĆ et al. 1983). Indeed, imaginary experiments are designed while planning a real experiment.

Yet, we think that NORTON (1996) has a point when citing J. Brown:

"A Platonic thought experiment is a single thought experiment which destroys an old or existing theory and simultaneously generates a new one; it is a priori in that it is not based on new empirical evidence nor is it merely logically derived from old data; and it is an advance in that the resulting theory is better than the predecessor theory."

Einstein in his profound thinking was partly influenced by Mach. But luckily not in that particular field, as he designed many thought, not imaginary, experiments. Indeed, before Einstein there were many imaginary experimenters, and very few thought experimenters - one of them was Galileo Galilei, for instance; now it is widely believed that he designed a thought experiment while he was working on his principle of inertia (without ever noticing it).

Later, when Newton formulated the principle of inertia, it lost its former connection to the thought experiment that was laid under it (NEWTON, 1964). This formulation shows that Newton treated inertia (vis insita) as something already accepted by the scientific community, and so he did not feel the need to prove the existence of it, nor, of course, to mention Galileo. But it didn't stop Newton from designing a bucket thought experiment. 


\section{THOUGHT EXPERIMENT - DEFINITIONS}

In the meantime thought experiment was defined by NorTON (1991), but to our opinion not on enough broad and sound bases. So, now we shall give a radically modified definition of a thought experiment, in order to obtain a useful criterion for distinguishing thought experiments from, say, presocratic imaginative thinking exercises.

In Einstein's paper (EINSTEIN, 1907) published in 1907, one can find the following passages:

“...Let us consider two referent systems $\Sigma_{1}$ and $\Sigma_{2}$. Let $\Sigma_{1}$ be accelerated along the direction of it's $x$-axis with acceleration (constant in time) equal to $\gamma$. Let us suppose that $\Sigma_{2}$ is at rest, but is located in the homogenous gravitational field, which is giving the acceleration $-\gamma$ in the direction of $x$-axis.

As is well known, physical laws in system $\Sigma_{1}$ are not different of those in system $\Sigma_{2}$; this is connected to the fact that in the gravitational field all material bodies are accelerated in the same manner..."

We have here, stated in a rather rudimental manner, the principle of equivalence, which played the fundamental role in forming the general theory of relativity. In year 1913, EINSTEIN, together with M. GROSSMANN, gave a more precise definition of this principle, stressing that the equivalency of the referent system in the gravitational field and accelerated referent system is strictly local.

What we are interested in, though, is the thought experiment that Einstein proposed ${ }^{1}$ in order to prove the principle of equivalence (one of thought experiments that could be found in almost every survey of the general theory of relativity):

Let us imagine an elevator (RESCHER, 1991) in Space, far from any gravitational mass whatsoever, and let this elevator be accelerated. If acceleration were constant and equal to $g$, a man inside it would feel as if he were in the gravitational field of the Earth, and vice versa, in the elevator that is in the state of free fall inside the Earth's gravitational field, acceleration obtained in this manner completely neutralizes the effect of Earth's gravity, introducing thus weightlessness.

The conclusion is exactly the same as the one from EINSTEIN's paper (1907); namely, the principle of equivalence is one of the basic principles of nature. But in the case of the elevator thought experiment, the conditions under which it is possible to identify the two aforementioned referent systems, are better defined (the size of the elevator is small compared to the size of the gravitating mass, thus the effect of tidal forces could be neglected; so the principle of equivalence could be applied only locally, the feature that is included in its general definition - MiSNER et al. 1973). Einstein was not the first one to come to the idea of checking Galileo's observation of the equivalence of inert and gravitational mass, in his papers he mentions Etövös's brilliant experimental treatment of the problem (MISNER et al. 1973). But Etövös, after his famous measurement of the difference of inert and gravitational mass, concluded only that they do not differ more then $10^{-9}$, while Einstein (RESCHER, 1991; EINSTEIN and GROSSMANN,1913) using his chest thought experiment [which is later to become the famous elevator thought experiment (EINSTEIN and INFELD 1938), concludes that inert and gravitational mass are identical, and draws from this conclusions about the equivalence of an accelerated coordinate system and a coordinate system that is located in the gravitational field giving the same acceleration, thus, bringing into the focus the curvature of space-time, and constructing on this foundations his general theory of relativity.

Of course, the situation described in the elevator thought experiment has been, mutatis mutandis, realized in the case of a space ship circling around the Earth (radial component of

\footnotetext{
${ }^{1}$ EINSTEIN himself liked to call this object a chest (EINSTEIN and GROSSMANN, 1913; RESCHER, 1991), until with INFELD they introduced an elevator (1938).
} 
its acceleration equals $g$, so the gravity inside the ship is absent, i.e. one has weightlessness), but the thought experiment is much more illustrative. As it can be said that experiments perform a scientific procedure, especially in a laboratory, to test a hypothesis or theories, it also could be said:

Thought experiments, as a subset of scientific experiments, are using real physical objects, and putting them into relations (governed by physical laws) which can not be realized at the present moment (i.e. using abstractions, but not at all independent of physical reality), or, if can be realized, it is much clearer and simpler to describe them by the help of a thought experiment. Yet thought experiments use well known concepts, gained from innumerous real experiments, which after the tedious analysis have been put into set of theorems, lemmas and corollaries, and extend those concepts to the cases that have never been realized before; thus enabling, by the unquestionable power of deduction, scientists to choose among various theories (or hypothesis) that are describing the phenomenon.

Thus working definition:

Thought experiments, using the existing knowledge of humankind, by exploiting real physical objects and imagined relations of them, put on test new cases which have never been scientifically described, or are easier described by the imaginative power of such experiments.

The "working definition" is, in fact, derived from many thought experiments, but, as already stressed; Einstein's elevator thought experiment is most intimately connected with it. Yet, to our opinion, it is more precise and clear than NORTON's definition (1991), part of which in the formulation of M. BISHOP (1999) states: "Thought experiments are arguments which: (i) posit hypothetical or counterfactual state of affairs..." There is nothing hypothetical, neither counterfactual about an elevator falling freely inside the Earth's gravitational field, it is just not so advisable to perform such a "state of affairs" in reality, or it was not until performed in the case of a space ship circling around the Earth.

Remembering the controversy following the train thought experiment which was proposed by Einstein (RESCHER, 1991) - the opinions going from claiming it an obvious mistake, to taking it as a full proof of non-existence of absolute simultaneity (for instance, BORN (1962), cautiously uses a barge and sound for the modified thought experiment of that kind), forced us to mention a few thoughts on "proving ability" of thought experiment.

Proving ability, of course, is pretty much different from being an argument as Norton (NORTON, 1991), and many others are claiming, and BISHOP (1999) is rightfully denying. It only has the same capacity real experiments possess, that is, the capacity to decide which kind of argumentation in different theories is correct.

\section{CLASSIFYING THOUGHT EXPERIMENTS}

After defining thought experiment, and explaining its proving ability, those experiments can be classified (BABOVIĆ et al. 1983) as follows:

\section{ACCORDING TO THEIR PURPOSE}

\section{Illustration of}

i. a part of the theory (twin-paradox, light-ray in an elevator moving far from gravitational fields) (BABOviĆ et al. 1983),

ii. the basic concepts of some theory (the elevator experiment) (RESCHER, 1991).

2. Proof of the part of some theory - or its basic concepts [the elevator experiment (RESCHER, 1991)]; that thought experiment, laid down in Einstein's book is treated by its designer as a definite proof of the principle of equivalence of inert and gravitational masses, distinguishing thus his point of view from Etövös's). 
3. Analyzing doubtful parts of some theory.

4. Helping in deriving formulae [obtaining (BABOviĆ et al. 1983) the time-dilatation formula, for instance].

\section{ACCORDING TO REALIZABILITY}

1. Unrealizable

i. momentarily (the elevator experiment before space era)

ii. permanently (light-ray in an elevator moving far from gravitational fields) (BABOvIĆ et al. 1983)

2. Realizable.

Of course, there are a lot of attempts to formulate the thought experiments that can not be realized in principle [counterfactual in the definition of NORTON (1991), RESCHER (1991), BISHOP (1999)], but since one has there contradictio in adjecto, those must be excluded from the set of thought experiments. For instance, perpetuum mobile is a concept that has been intriguing to human mind throughout the history of mankind, but since it violates the first and second law of thermodynamics, projects of it can not be considered thought experiments.

On the other hand, the elevator thought experiment was not realizable when proposed, but is realizable in principle, and now is performed daily by every satellite circling around the Earth. Yet Einstein's childhood idea of traveling with the ray of light in principle is not realizable, because nothing that has a rest mass can travel with the velocity of light, and so that situation can not be regarded as a thought experiment at all (but, of course, Einstein proposed such ideas before discovering his special theory - it was, so to speak, merely a child's flight of fantasy, but one that had the profound influence on the young scientist's mind. So the mistake was made by accident, which can not be said for the train experiment, or some other imaginary performances that follow).

Bertrand Russell, one of the twentieth century most renowned logicians, in his famous book about the theory of relativity (RUSSEL, 1985) gave an example of relativistic addition of velocities, which is indeed very illustrative, but incorrect. Namely, he uses in his explanation an escalator which is moving with the velocity of light, and that is, of course, impossible in principle. Furthermore, it was recently pointed out to one very widespread mistake which is based on attaching a coordinate system to a light ray (RUSSEL, 1985). This "operation" (very similar to Einstein's childhood idea of traveling on the light ray) could be even thought of as violation of the principle of constancy of the velocity of light. Namely, in the referent system that moves with the light ray the light would have zero velocity, in obvious contradiction with this famous principle. But, in order to attach something to anything else, those objects have to be materialized, even in a thought experiment. Thus, such a referent system has to become something more solid than mere four lines in space-time (as drawn on the blackboard), i.e. it has to be realized by one or more material objects, whose relations could offer an opportunity of determining the distances and time intervals. Yet, material bodies have the rest mass different from zero, and according to the special theory of relativity such objects can not travel with the velocity of light. It means that, in principle, no referent system could be attached to a light ray. So, the aforementioned operation is not a thought experiment at all.

Still, in order to formulate his principle of inertia, Galileo had to make some abstractions (but strictly governed by physical laws): for instance, to imagine the movement without friction, which is not exactly impossible, but is very hard to realize. Nevertheless, since such abstractions are at the very core of thought experimenting, see our definition, this can be considered a thought experiment. Yet in the case of Zeno's paradox things are different. Achilles, since motion is not illusory, will catch up with the tortoise, so Zeno's paradox states something that is impossible in principle and therefore is not a thought experiment. This is not to be understood as a naïve try to resolve Zeno's sophisticated 
example, but only to exclude it from the set of thought experiments (stated differently: philosophy is not an experimental science).

\section{FINAL REMARKS}

Even though Einstein has made thought experiments famous, he was not the first one to use them. He didn't even coin the term. Yet lot of researchers regard him as the one of the greatest thought experimenters ever, and there are some who are inclined to attribute him all the credit for thought experiments in physics.

We also consider Einstein the greatest thought experimenter, but do not deny that the term existed before Einstein, and that there are many others who designed the significant thought experiments (Heisenberg, Bohr, not being among the least).

Also, there are many researchers who believe that Galileo designed thought experiments and some who even pronounce certain presocratic thinking for thought experiments. Yet, in our opinion, thought experiments are subset of real experiments and they are basically connected to experimental sciences (physics, before all) so one should not accept every imaginative application for a thought experiment. That is the reason why new working definition of a thought experiment is being offered, underlying the proving ability of thought experiments, to the measure there is proving ability in real experiments. Applying these definitions some undecided examples are properly classified. For instance, in order to discover the principle of inertia Galileo needed to make some abstractions, as if there were cases of movement without friction, so this could be considered, according to aforementioned definition, a thought experiment. But it is not the case with Greek thinkers because, to state it explicitly and shortly, philosophy is not an experimental science.

Thought experiments generate their results so elegantly, that there is scarcely a more effective device available to the scientist. Importance of thought experiments can be seen in Einstein's work, because he used thought experiments in a direct form in his papers, both for proving basic principles (lift thought experiment, Einstein, 1913), and proving parts of the theory, NORTON 1991).

\section{Acknowledgment}

This work was supported in part by the Ministry of Science, Republic of Serbia (project No. 171021).

\section{References}

[1] Babović, V., Ristić, V., Todorović, D., Thought Experiment in Physics, Coll. of Sci. Papers of Fac. of Sci. Kragujevac 4, (1983) 7-29 [in Serbian].

[2] Bishop, M., Why Thought Experiments Are Not Arguments, Philosophy of Science 66, (1999) 534-541.

[3] Born, M., Einstein's Theory of Relativity, Dover Publications Inc., New York, (1962), pp. 227-229.

[4] EINSTEIN, A., Über das Relatvitätsprinzip und die aus demselben gezongenen Folgerungen, Jahrb. d. Radioaktivität u. Elektronik, Bd. 4 (1907) 411-462. 
[5] EINSTEIN, A., Über die spezielle und die algemeine Relativitätstheorie, Vieweg, Bhraunschweig (1917), translated by Lawson, R.W. Relativity: The Special and General Theory, Methuen \& Co Ltd., London (1920), p. 25, p. 60.

[6] Einstein A., Grossmann, M., Entwurf einer verallgemeinerten Relatvitätstheorie und Theorie der Gravitation, Zs. Math. und Phys. 62, (1913) 225.

[7] Einstein, A., Infeld, L., The Evolution of Physics, Cambridge University Press (1938).

[8] Misner, C.W., Thorne, K.S., Wheeler, J.A., Gravitation, W.H. Freemann and Co., San Francisco (1973), pp. 386.

[9] Newton, I. The Mathematical Principles of Natural Philosophy, Philosophical Library, New York (1964), p. 13.

[10] Norton, J., Thought experiments in Einstein's work, in: Horowitz, T., Massey, G. (eds.) Thought Experiments in Science and Philosophy, Rowman \& Litlefield Publishers (1991) pp. 129-148

[11] Norton, J., Are Thought Experiments just what you thought?, Canadian Journal of Philosophy 23 (3) (1996) 333-336.

[12] Rescher, N., Thought experiments in Presocratic Philosophy, in: Horowitz, T., Massey, G. (eds.) Thought Experiments in Science and Philosophy, Rowman \& Litlefield Publishers (1991) pp. 31-42.

[13] Ristić, V., Radulović, M., Thought Experiment and Computer Experiment Similarities and Differences, Kragujevac J. Sci. 23, (2001) 47-50.

[14] Russel, B., ABC of Relativity, Routledge, London (1985), p. 31.

[15] SorEnsen, R., Thought Experiments, Oxford University Press (1992) pp. 8-15.

[16] Thought experiment on Wikipedia, http://en.wikipedia.org/wiki/Thought_experiment 